\title{
Nutrient generation and retrieval from the host cell cytosol by intra-vacuolar Legionella pneumophila
}

\author{
Christopher T. D. Price, Ashley M. Richards and Yousef Abu Kwaik* \\ Department of Microbiology and Immunology and Center for Predictive Medicine, College of Medicine, University of Louisville, Louisville, KY, USA \\ ${ }^{*}$ Correspondence: abukwaik@louisville.edu
}

Edited by:

Wolfgang Eisenreich, Technische Universität München, Germany

Reviewed by:

Klaus Heuner, Robert Koch-Institute, Germany

Keywords: Legionnaires' disease, proteasomes, ubiquitin, slc transporter, AnkB, F-box, farnesylation

Microbial acquisition of nutrients in vivo is a fundamental aspect of infectious diseases, and is a potential target for antimicrobial therapy. Part of the innate host defense against microbial infection is nutritional restriction of access to sources of host nutrients (Abu Kwaik and Bumann, 2013; Eisenreich et al., 2013). Despite this host nutritional restriction, there has been a long held presumption that the host cell cytosol has sufficient nutrients for any intracellular pathogen, although many bacteria fail to grow in the host cytosol if they are microinjected (Goetz et al., 2001). However, recent studies on the two intra-vacuolar pathogens Anaplasma phagocytophilum (Niu et al., 2012) and Legionella pneumophila (Price et al., 2011) and the cytosolic pathogen Francisella tularensis (Steele et al., 2013) have clearly shown that the levels of amino acids in the host cell cytosol are below the threshold sufficient to meet the tremendous demands for carbon, nitrogen and energy to power the robust intracellular proliferation of these pathogens (Abu Kwaik and Bumann, 2013). Therefore, these intracellular pathogens have evolved with efficient strategies to boost the levels of host amino acids to meet their demands for higher levels of carbon, nitrogen and energy sources (Abu Kwaik and Bumann, 2013; Fonseca and Swanson, 2014). There is an emerging paradigm of specific microbial strategies that directly trigger the host cell to boost the cellular levels of essential microbial nutrients, and this paradigm has been designated as "nutritional virulence" (Abu Kwaik and Bumann, 2013). This opinion article is focused on nutritional virulence of L. pneumophila.
In the aquatic environment, L. pneumophila proliferates within protozoa, which impact bacterial ecology and pathogenicity (Al-Quadan et al., 2012). Upon transmission to humans, L. pneumophila proliferates in alveolar macrophages within the Legionellacontaining vacuole (LCV) that is ER-derived and evades lysosomal fusion (Figure 1). Within both evolutionarily distant host cells, the Dot/Icm type IV secretion system of L. pneumophila injects $\sim 300$ protein effectors (Zhu et al., 2011; Luo, 2011a) that govern biogenesis of the LCV and modulate a myriad of cellular processes to enable intra-vacuolar proliferation (Figure 1) (Luo, 2011b; Richards et al., 2013).

Amino acids are the main sources of carbon, nitrogen and energy for L. pneumophila, which metabolizes them through the TCA cycle (Pine et al., 1979), but also metabolizes minor amounts of glucose in vitro using the Entner-Doudoroff pathway (Eylert et al., 2010; Price et al., 2011). Although L. pneumophila utilizes amino acids as the main sources of carbon and energy, the pathogen is auxotrophic for seven amino acids (Cys, Met, Arg, Thr, Val, Ile, and Leu) (Eylert et al., 2010; Price et al., 2014). Remarkably, there is a high level of synchronization in amino acids auxotrophy between L. pneumophila and its host cells, which has likely played a factor in nutritional evolution of L. pneumophila as an intra-vacuolar pathogen (Price et al., 2014).

Interestingly, intra-vacuolar L. pneumophila up-regulates its own amino acids transporters, indicating increased demands for amino acids in the intra-vacuolar environment (Bruggemann et al., 2006; Faucher et al., 2011; Eisenreich et al., 2013). Since the generation time of intra-vacuolar L. pneumophila is $\sim 40 \mathrm{~min}$, this organism requires high levels of amino acids to be imported from the host cytosol into the LCV lumen (Schunder et al., 2014). A long-held presumption has been that the host cell cytosol is rich in nutrients for invading pathogens.

However, recent studies clearly indicate that the basal levels of host cellular amino acids are below the threshold sufficient for the robust intra-vacuolar proliferation of L. pneumophila (Sauer et al., 2005; Wieland et al., 2005) To achieve that needed threshold, L. pneumophila promotes host proteasomal degradation (Price et al., 2011) of LCV-decorated polyubiquitinated proteins (Dorer et al., 2006; Price et al., 2009, 2011; Lomma et al., 2010) mediated by the AnkB effector.

Within human macrophages and amoeba, the AnkB translocated effector of L. pneumophila strain AA100/130b is localized to the cytosolic face of the LCV membrane through host-mediated farnesylation of its C-terminal CaaX motif (Figure 1) (Price et al., 2010; AlQuadan et al., 2011; Al-Quadan and Kwaik, 2011). On the LCV membrane, AnkB interacts with the host SCF1 ubiquitin ligase (Figure 1) (Bruckert et al., 2014). As a bona fide F-box effector (Ensminger and Isberg, 2010; Lomma et al., 2010; Price and Abu Kwaik, 2010), AnkB triggers decoration of the LCV with Lys $^{48}$-linked polyubiquitinated proteins that are targeted for proteasomal degradation (Figure 1) (Price et al., 2011). The metabolomic profile 


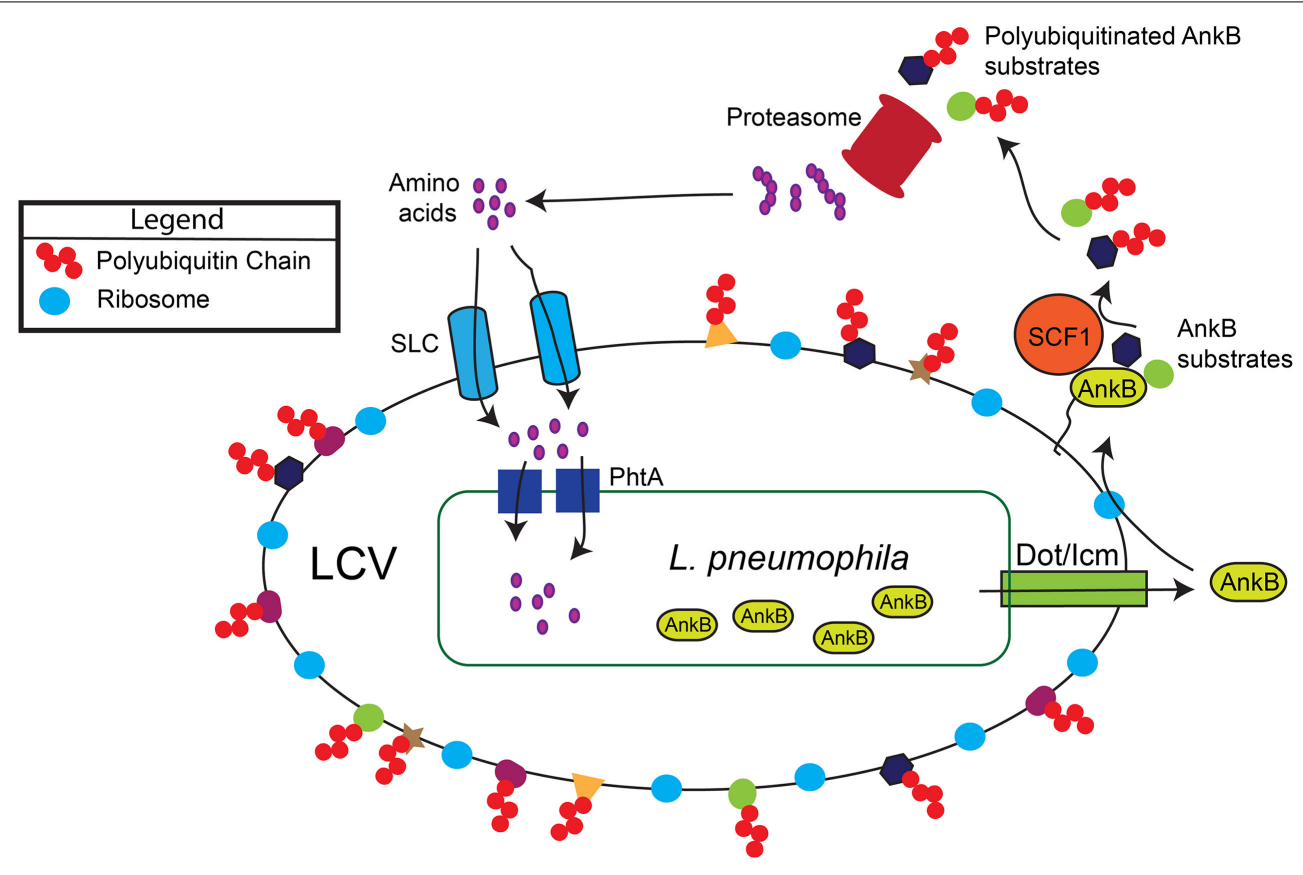

FIGURE 1 | Generation of a surplus of host amino acids by L. pneumophila and their import into the LCV. The AnkB effector is translocated into macrophages and amoebae by the Dot/lcm type IV secretion system of $L$. pneumophila. AnkB is immediately farnesylated and anchored into the cytosolic face of the LCV membrane where it interacts with the eukaryotic SCF1 ubiquitin ligase complex. The AnkB effector functions as a platform for the docking of $\mathrm{Lys}^{48}$-linked polyubiquitinated proteins to the
LCV that are subsequently degraded by the proteasome, which generates a surplus of cellular amino acids above the threshold necessary for intra-vacuolar proliferation. The amino acids are likely imported into the LCV through various host SLC amino acid transporters in the LCV membrane, but the identity of the transporters is still to be determined. The amino acids are acquired by $L$. pneumophila through numerous $A B C$ transporters and amino acid permeases such as the threonine transporter PhtA. of L. pneumophila-infected amoeba and human cells have shown an AnkBdependent dramatic rise in the levels of all cellular amino acids (Price et al., 2011), and this is initiated rapidly upon bacterial attachment to the macrophage plasma membrane (Bruckert et al., 2014). Importantly, inhibition of host proteasomal degradation abolishes intracellular proliferation of L. pneumophila strains AA100/130b and Philadelphia (Dorer et al., 2006; Price et al., 2011). The L. pneumophila-generated surplus of host cell amino acids may explain the lack of an intracellular defect for the lysine and tryptophan auxotrophic mutants of L. pneumophila (Mintz et al., 1988; Ensminger et al., 2012).

Loss of AnkB in two independent isolates (AA100 and Paris) results in varying degrees of failure in intra-vacuolar proliferation and attenuation in the mouse model of Legionnaires' disease (AlKhodor et al., 2008; Lomma et al., 2010). These defects for the AA100 strain are totally overcome upon supplementation of a mixture of amino acids in macrophages, amoeba and in the mouse model, similar to genetic complementation (Price et al., 2011). Importantly, silencing of the host SCF1 ubiquitin ligase, interference with $\mathrm{Lys}^{48}$-linked polyubiquitination, or inhibition of the host proteasomes block intra-vacuolar proliferation of L. pneumophila, but the block is relieved upon supplementation of an excess mixture of amino acids (Price et al., 2009, 2011). Surprisingly, the intra-vacuolar proliferation defect of the $a n k B$ mutant is rescued by many individual amino acids, such as Cys, Ala or Ser, which are essential or metabolically favorable for L. pneumophila (Pine et al., 1979). Interestingly, although Gln is the most abundant amino acid in human cells, supplementation of infected hMDMs with excess Gln alone efficiently rescues the ankB mutant (Price et al., 2011), while Glu is a major source of carbon and energy in vitro (Pine et al., 1979). These findings indicate that the basal levels of cellular amino acids are below the threshold sufficient for intra-vacuolar proliferation of L. pneumophila.

Remarkably, pyruvate or citrate supplementation is as effective as amino acids in rescuing the intra-vacuolar growth defect of the ankB mutant, which indicates that the LCV is capable of importing these two substrates that can feed the TCA cycle, in addition to the documented reliance of intra-vacuolar L. pneumophila on amino acids (Schunder et al., 2014). In addition, L. pneumophila utilizes glucose through the Entner-Doudoroff pathway, which is required for proliferation within the amoeba host (Eylert et al., 2010). During inflammation, macrophages undergo upregulation of glucose uptake and anaerobic glycolysis (Warburg-effect), which generates additional pyruvate (Eisenreich et al., 2013), and it is likely that both glucose and pyruvate are imported by the LCV. In addition, L. pneumophila-infected macrophages exhibit a pro-inflammatory phenotype. Taken together, it is likely that a multi-prong nutritional virulence strategy is utilized by L. pneumophila to 
generate and retrieve a diversified portfolio of sources of carbon and energy from the host cell. The host solute carrier (SLC) family of membrane proteins (Cedernaes et al., 2011; Schioth et al., 2013) that transport various compounds, including amino acids, TCA intermediates, glucose, lipids, and drugs are likely to be involved in import of various compounds by the LCV membrane (Figure 1) (Wieland et al., 2005). Future studies should unravel the host metabolites and the mechanism of their import into the LCV lumen, and subsequently by the bacterial membrane. Deciphering microbial nutrition and metabolism in vivo is essential for our understanding of hostmicrobe interaction, and nutrient retrieval strategies by intracellular pathogens are potential targets for therapy.

\section{ACKNOWLEDGMENTS}

Yousef Abu Kwaik is supported by Public Health Service Awards R01AI069321 and R21AI107978 from NIAID and by the commonwealth of Kentucky Research Challenge Trust Fund.

\section{REFERENCES}

Abu Kwaik, Y., and Bumann, D. (2013). Microbial quest for food in vivo: 'nutritional virulence' as an emerging paradigm. Cell. Microbiol. 15, 882-890. doi: $10.1111 / \mathrm{cmi} .12138$

Al-Khodor, S., Price, C. T., Habyarimana, F., Kalia, A., and Abu Kwaik, Y. (2008). A Dot/Icmtranslocated ankyrin protein of Legionella pneumophila is required for intracellular proliferation within human macrophages and protozoa. Mol. Microbiol. 70, 908-923. doi: 10.1111/j.13652958.2008.06453.x

Al-Quadan, T., and Kwaik, Y. A. (2011). Molecular characterization of exploitation of the Polyubiquitination and Farnesylation Machineries of Dictyostelium discoideum by the AnkB F-box effector of Legionella pneumophila. Front. Microbiol. 2:23. doi: 10.3389/fmicb.2011. 00023

Al-Quadan, T., Price, C. T., and Abu Kwaik, Y. (2012). Exploitation of evolutionarily conserved amoeba and mammalian processes by Legionella. Trends Microbiol. 20, 299-306. doi: 10.1016/j.tim.2012. 03.005

Al-Quadan, T., Price, C. T., London, N., SchuelerFurman, O., and AbuKwaik, Y. (2011). Anchoring of bacterial effectors to host membranes through host-mediated lipidation by prenylation: a common paradigm. Trends Microbiol. 19, 573-579. doi: 10.1016/j.tim.2011.08.003

Bruckert, W. M., Price, C. T., and Abu Kwaik, Y. (2014). Rapid nutritional remodeling of the host cell upon attachment of Legionella pneumophila. Infect. Immun. 82, 72-82. doi: 10.1128/IAI. 01079-13
Bruggemann, H., Hagman, A., Jules, M., Sismeiro, O., Dillies, M. A., Gouyette, C., et al. (2006). Virulence strategies for infecting phagocytes deduced from the in vivo transcriptional program of Legionella pneumophila. Cell. Microbiol. 8, 1228-1240. doi: 10.1111/j.1462-5822.2006.00703.x

Cedernaes, J., Olszewski, P. K., Almen, M. S., Stephansson, O., Levine, A. S., Fredriksson, R., et al. (2011). Comprehensive analysis of localization of 78 solute carrier genes throughout the subsections of the rat gastrointestinal tract. Biochem. Biophys. Res. Commun. 411, 702-707. doi: 10.1016/j.bbrc.2011.07.005

Dorer, M. S., Kirton, D., Bader, J. S., and Isberg, R. R. (2006). RNA interference analysis of Legionella in Drosophila cells: exploitation of early secretory apparatus dynamics. PLoS Pathog. 2:e34. doi: 10. 1371/journal.ppat.0020034

Eisenreich, W., Heesemann, J., Rudel, T., and Goebel, W. (2013). Metabolic host responses to infection by intracellular bacterial pathogens. Front. Cell. Infect. Microbiol. 3:24. doi: 10.3389/fcimb.2013. 00024

Ensminger, A. W., and Isberg, R. R. (2010). E3 ubiquitin ligase activity and targeting of BAT3 by multiple Legionella pneumophila translocated substrates. Infect. Immun. 78, 3905-3919. doi: 10.1128/IAI. 00344-10

Ensminger, A. W., Yassin, Y., Miron, A., and Isberg, R. R. (2012). Experimental evolution of Legionella pneumophila in mouse macrophages leads to strains with altered determinants of environmental survival. PLoS Pathog. 8:e1002731. doi: 10.1371/journal.ppat.1002731

Eylert, E., Herrmann, V., Jules, M., Gillmaier, N., Lautner, M., Buchrieser, C., et al. (2010). Isotopologue profiling of Legionella pneumophila: role of serine and glucose as carbon substrates. J. Biol. Chem. 285, 22232-22243. doi: 10.1074/jbc.M110.128678

Faucher, S. P., Mueller, C. A., and Shuman, H. A. (2011). Legionella pneumophila transcriptome during intracellular multiplication in human macrophages. Front. Microbiol. 2:60. doi: 10.3389/ fmicb.2011.00060

Fonseca, M. V., and Swanson, M. S. (2014). Nutrient salvaging and metabolism by the intracellular pathogen Legionella pneumophila. Front. Cell. Infect. Microbiol. 4:12. doi: 10.3389/fcimb.2014.00012

Goetz, M., Bubert, A., Wang, G., Chico-Calero, I., Vazquez-Boland, J. A., Beck, M., et al. (2001). Microinjection and growth of bacteria in the cytosol of mammalian host cells. Proc. Natl. Acad. Sci. U.S.A. 98, 12221-12226. doi: 10.1073/pnas.211106398

Lomma, M., Dervins-Ravault, D., Rolando, M., Nora, T., Newton, H. J., Sansom, F. M., et al. (2010). The Legionella pneumophila F-box protein Lpp2082 (AnkB) modulates ubiquitination of the host protein parvin B and promotes intracellular replication. Cell. Microbiol. 12, 1272-1291. doi: 10.1111/j.1462-5822.2010.01467.x

Luo, Z. Q. (2011a). Targeting one of its own: expanding roles of substrates of the Legionella pneumophila Dot/Icm Type IV secretion system. Front. Microbiol. 2:31. doi: 10.3389/fmicb.2011.00031

Luo, Z. Q. (2011b). Striking a balance: modulation of host cell death pathways by Legionella pneumophila. Front. Microbiol. 2:36. doi: 10.3389/fmicb.2011.00036

Mintz, C. S., Chen, J. X., and Shuman, H. A. (1988). Isolation and characterization of auxotrophic mutants of Legionella pneumophila that fail to multiply in human monocytes. Infect. Immun.. 56, 1449-1455.

Niu, H., Xiong, Q., Yamamoto, A., Hayashi-Nishino, M., and Rikihisa, Y. (2012). Autophagosomes induced by a bacterial Beclin 1 binding protein facilitate obligatory intracellular infection. Proc. Natl. Acad. Sci. U.S.A. 109, 20800-20807. doi: 10.1073/pnas.1218674109

Pine, L., George, J. R., Reeves, M. W., and Harrell, W. K. (1979). Development of a chemically defined liquid medium for growth of Legionella pneumophila. J. Clin. Microbiol. 9, 615-626.

Price, C. T., and Abu Kwaik, Y. (2010). Exploitation of host polyubiquitination machinery through molecular mimicry by Eukaryotic-like bacterial F-box effectors. Front. Microbiol. 1, 122. doi: 10.3389/fmicb.2010.00122

Price, C. T., Al-Khodor, S., Al-Quadan, T., Santic, M., Habyarimana, F., Kalia, A., et al. (2009). Molecular mimicry by an F-box effector of Legionella pneumophila hijacks a conserved polyubiquitination machinery within macrophages and protozoa. PLoS Pathog. 5:e1000704. doi: 10.1371/journal.ppat. 1000704

Price, C. T., Al-Quadan, T., Santic, M., Jones, S. C., and Abu Kwaik, Y. (2010). Exploitation of conserved eukaryotic host cell farnesylation machinery by an F-box effector of Legionella pneumophila. J. Exp. Med. 207, 1713-1726. doi: 10.1084/jem.20100771

Price, C. T., Al-Quadan, T., Santic, M., Rosenshine, I., and Abu Kwaik, Y. (2011). Host proteasomal degradation generates amino acids essential for intracellular bacterial growth. Science 334, 1553-1557. doi: 10.1126/science. 1212868

Price, C. T., Richards, A. M., Von Dwingelo, J. E., Samara, H. A., and Abu Kwaik, Y. (2014). Amoeba host-Legionella synchronization of amino acid auxotrophy and its role in bacterial adaptation and pathogenic evolution. Environ. Microbiol. 16, 350-358. doi: 10.1111/1462-2920.12290

Richards, A. M., Von Dwingelo, J. E., Price, C. T., and Abu Kwaik, Y. (2013). Cellular microbiology and molecular ecology of Legionella-amoeba interaction. Virulence 4, 307-314. doi: 10.4161/viru.24290

Sauer, J. D., Bachman, M. A., and Swanson, M. S. (2005). The phagosomal transporter A couples threonine acquisition to differentiation and replication of Legionella pneumophila in macrophages. Proc. Natl. Acad. Sci. U.S.A. 102, 9924-9929. doi: 10.1073/pnas.0502767102

Schioth, H. B., Roshanbin, S., Hagglund, M. G., and Fredriksson, R. (2013). Evolutionary origin of amino acid transporter families SLC32, SLC36 and SLC38 and physiological, pathological and therapeutic aspects. Mol. Aspects Med. 34, 571-585. doi: 10.1016/j.mam.2012.07.012

Schunder, E., Gillmaier, N., Kutzner, E., Herrmann, V., Lautner, M., Heuner, K., et al. (2014). Amino acid uptake and metabolism of Legionella pneumophila hosted by Acanthamoeba castellanii. J. Biol. Chem. 289, 21040-21054. doi: 10.1074/jbc.M114.570085

Steele, S., Brunton, J., Ziehr, B., Taft-Benz, S., Moorman, N., and Kawula, T. (2013) 
Francisella tularensis harvests nutrients derived via ATG5-Independent autophagy to support intracellular growth. PLoS Pathog. 9:e1003562. doi: 10.1371/journal.ppat.1003562

Wieland, H., Ullrich, S., Lang, F., and Neumeister, B. (2005). Intracellular multiplication of Legionella pneumophila depends on host cell amino acid transporter SLC1A5. Mol. Microbiol. 55, 1528-1537. doi: 10.1111/j.1365-2958.2005. 04490.x

Zhu, W., Banga, S., Tan, Y., Zheng, C., Stephenson, R., Gately, J., et al. (2011). Comprehensive identification of protein substrates of the Dot/Icm type IV transporter of Legionella pneumophila.
PLoS ONE 6:e17638. doi: 10.1371/journal.pone. 0017638

Conflict of Interest Statement: The authors declare that the research was conducted in the absence of any commercial or financial relationships that could be construed as a potential conflict of interest.

Received: 18 July 2014; paper pending published: 24 July 2014; accepted: 27 July 2014; published online: 26 August 2014.

Citation: Price CTD, Richards AM and Abu Kwaik Y (2014) Nutrient generation and retrieval from the host cell cytosol by intra-vacuolar Legionella pneumophila.
Front. Cell. Infect. Microbiol. 4:111. doi: 10.3389/fcimb. 2014.00111

This article was submitted to the journal Frontiers in Cellular and Infection Microbiology.

Copyright (c) 2014 Price, Richards and Abu Kwaik. This is an open-access article distributed under the terms of the Creative Commons Attribution License (CC BY). The use, distribution or reproduction in other forums is permitted, provided the original author(s) or licensor are credited and that the original publication in this journal is cited, in accordance with accepted academic practice. No use, distribution or reproduction is permitted which does not comply with these terms. 